OPEN ACCESS

Edited by:

Pasquale Martino,

Santa Maria Hospital, Italy

Reviewed by:

Pietro Pepe,

Cannizzaro Hospital, Italy

Alessandro Stefano,

Institute of Bioimaging and Molecular

Physiology (CNR), Italy

*Correspondence:

Alfonso Reginelli

alfonsoreginell@hotmail.com

Specialty section:

This article was submitted to

Cancer Imaging and

Image-directed Interventions,

a section of the journal

Frontiers in Oncology

Received: 29 October 2021 Accepted: 29 November 2021 Published: 21 December 2021

Citation:

Urraro $F$, Nardone $V$, Reginelli $A$,

Varelli C, Angrisani A, Patanè $V$,

D’Ambrosio L, Roccatagliata P,

Russo GM, Gallo L, De Chiara M,

Altucci L and Cappabianca S (2021)

MRI Radiomics in Prostate

Cancer: A Reliability Study.

Front. Oncol. 11:805137.

doi: $10.3389 /$ fonc. 2021.805137

\title{
MRI Radiomics in Prostate Cancer: A Reliability Study
}

\begin{abstract}
Fabrizio Urraro ${ }^{1}$, Valerio Nardone ${ }^{1}$, Alfonso Reginelli ${ }^{1 *}$, Carlo Varelli ${ }^{2}$, Antonio Angrisani ${ }^{1}$, Vittorio Patanè ', Luca D'Ambrosio ${ }^{1}$, Pietro Roccatagliata ${ }^{1}$, Gaetano Maria Russo ${ }^{1}$, Luigi Gallo ${ }^{1}$, Marco De Chiara ${ }^{1}$, Lucia Altucci ${ }^{1}$ and Salvatore Cappabianca ${ }^{1}$
\end{abstract}

${ }^{1}$ Department of Precision Medicine, University of Campania Luigi Vanvitelli, Naples, Italy, ${ }^{2}$ Istituto Diagnostico Varelli, Naples, Italy

Background: Radiomics can provide quantitative features from medical imaging that can be correlated to clinical endpoints. The challenges relevant to robustness of radiomics features have been analyzed by many researchers, as it seems to be influenced by acquisition and reconstruction protocols, as well as by the segmentation of the region of interest (ROI). Prostate cancer ( $\mathrm{PCa}$ ) represents a difficult playground for this technique, due to discrepancies in the identification of the cancer lesion and the heterogeneity of the acquisition protocols. The aim of this study was to investigate the reliability of radiomics in PCa magnetic resonance imaging (MRI).

Methods: A homogeneous cohort of patients with a PSA rise that underwent multiparametric MRI imaging of the prostate before biopsy was tested in this study. All the patients were acquired with the same MRI scanner, with a standardized protocol. The identification and the contouring of the region of interest (ROI) of an MRI suspicious cancer lesion were done by two radiologists with great experience in prostate cancer (>10 years). After the segmentation, the texture features were extracted with LIFEx. Texture features were then tested with intraclass coefficient correlation (ICC) analysis to analyze the reliability of the segmentation.

Results: Forty-four consecutive patients were included in the present analysis. In 26 patients (59.1\%), the prostate biopsy confirmed the presence of prostate cancer, which was scored as Gleason 6 in 6 patients (13.6\%), Gleason $3+4$ in 8 patients (18.2\%), and Gleason $4+3$ in 12 patients (27.3\%). The reliability analysis conversely showed poor reliability in the majority of the MRI acquisition (61\% in T2, 89\% in DWI50, 44\% in DWI400, and $83 \%$ in DWl1,500), with ADC acquisition only showing better reliability (poor reliability in only $33 \%$ of the texture features).

Conclusions: The low ratio of reliability in a monoinstitutional homogeneous cohort represents a significant alarm bell for the application of MRI radiomics in the field of prostate cancer. More work is needed in a clinical setting to further study the potential of $\mathrm{MRI}$ radiomics in prostate cancer.

Keywords: prostate cancer, radiomics, texture, target therapy, magnetic resonance imaging (MRI) 


\section{BACKGROUND}

Prostate cancer $(\mathrm{PCa})$ is the most frequent male malignancy with 1.4 million new diagnoses per year worldwide (1), and it represents the sixth leading cause of cancer death in men (2).

A pivotal role in clinical workup for PCa patients is played by pathology through prostate biopsy $(3,4)$ and the Gleason score assessment. The importance of tissue examination was recently proven when, among the other prognostic factors, tumor heterogeneity defined through genomic analyses showed to directly impact on overall survival and cancer control (5-8). Today, through biopsy and molecular assays, we can assess lesion molecular pattern that is more and more important given that PCa may show through several clinical scenarios. In fact, the clinical presentation of prostate cancer can range from a slow, localized, and indolent disease to a rapidly evolving lethal metastatic disease (9).

Adopting radiomics as an available and cost-effective tool, longitudinal monitoring as well as whole tumor examination is possible (e.g., to assist diagnostic random biopsies when sampling errors are likely to occur, due to intratumoral heterogeneity). The widespread use of medical imaging and the increasingly unleashed potential of radiomic features (RF) have made non-invasive examination as important as the invasive one (which still remains the gold standard to get a proper diagnosis of malignancy). Given its current role, radiomics, as a quantitative data extraction method, has received high expectations to become the new frontier of precision medicine and clinical imaging. With large image datasets and a "population-imaging" approach, RF may also be used to discover previously unknown markers and pattern of disease evolution, progression, and treatment response (10-13). Because of the discrepancies in identifying cancer lesions radiographically and within the various acquisition protocols, $\mathrm{PCa}$ represents a difficult playground for radiomics although RF could be truly helpful in many fields. For instance, radiomics could be applied for tumor localization and detection, as well as for prediction of prognosis, esteemed for a successful treatment (14, 15), or it could be used with follow-up imaging and combined with preintervention data to stratify the risk of patients for a personalized medicine approach.

The aim of this study was to investigate the reliability of radiomics, focusing on the robustness of radiomic features, in prostate cancer detection.

\section{METHODS}

\section{Population}

For the present study, we retrospectively evaluated a homogeneous cohort of consecutive patients with a PSA rise that underwent multiparametric MRI imaging of the prostate before biopsy in a defined time period from July 2017 to March 2019. All the patients subsequently underwent a random prostate biopsy and the presence of prostate cancer, as well as the Gleason score, was retrospectively collected.

\section{Magnetic Resonance Imaging Technical Protocol}

Every patient underwent multiparametric MRI (mpMRI) of the prostate. The MRI equipment and imaging protocols were made as described in the Prostate Imaging-Reporting and Data System (PI-RADS) version 2.1. MRI was performed on a 1.5-T scanner (MAGNETOM Aera ${ }^{\circledR}$; Siemens Healthcare, Erlangen, Germany), using a dedicated 16-channel phased-array body coil (Siemens Healthcare). Multiplanar (axial, coronal, and sagittal) T2-weighted (T2W) turbo spin-echo ( $Z 263 \mathrm{~mm} ; X$ $350 \mathrm{~mm}$; $Y 350 \mathrm{~mm}$; voxel size $0.6 \times 0.6 \times 3.5 \mathrm{~mm}$ ) and diffusionweighted MRI ( $Z 193 \mathrm{~mm}$; X $200 \mathrm{~mm}$; Y $77 \mathrm{~mm}$; voxel size $1.8 \times$ $1.8 \times 3.5 \mathrm{~mm}$ ) were performed with a single-shot echoplanar imaging sequence ( $b$-value $50 ; 800$ and $1,500 \mathrm{~s} / \mathrm{mm}^{2}$ ). The image software automatically calculated apparent diffusion coefficient (ADC) maps (Z $193 \mathrm{~mm}$; X $200 \mathrm{~mm}$; Y $77 \mathrm{~mm}$; voxel size $1.8 \times$ $1.8 \times 3.5 \mathrm{~mm}$ ). Dynamic contrast-enhanced (DCE) MRI was conducted using a 3D axial gradient echo sequence. Acquisitions were obtained before and after the administration of gadoliniumbased contrast medium (gadobutrol Gadovist ${ }^{\circledR}$ 1.0; Bayer Schering Pharma AG, Berlin, Germany) using a dose of 0.1 mmol $\mathrm{kg}$ at $1 \mathrm{ml} \mathrm{s}^{-1}$, using an automated injector (Ulrich Medical, Ulm, Germany). After the dynamic series, image subtraction of the contrast-enhanced images from the images before the administration of the contrast agent was performed.

\section{Feature Extraction}

The identification of an MRI suspicious target lesion was done by two radiologists with great experience in prostate cancer $(>10$ years) (see Figure 1).

After the identification, the regions of interest (ROI) were contoured by the same specialists blinded to each other on all the slides where the target lesion was visible. Both the segmentation of the ROI and the extraction of texture features were performed with the freeware software LIFEx $^{\odot}$ (16). This commercial software is able to extract several features coming from the gray-level histogram, shape, and four matrices of higher order statistics (GLCM, NGLDM, GLRLM, GLZLM) (see Table 1).

\section{Endpoints and Statistical Analysis}

Our data consist of the repetition of measurements of texture features for each target lesion identified on MRI imaging. As we want to test the reproducibility and the repeatability of texture features, with different operators, we decided to use intraclass coefficient correlation (ICC) as it is recognized as a method that is independent of the actual scale of measurement and of the size of the error that is considered acceptable (17).

We scored ICC values less than 0.5 as poor reliability, values between 0.5 and 0.75 as moderate reliability, values between 0.75 and 0.9 as good reliability, and values greater than 0.90 as excellent reliability (18-21). ICC estimates and their 95\% confidence intervals were based on a mean rating, absolute agreement, two-way mixed-effects model.

The distributions of ICC scores across the different classes of texture features and across the different sequences of mpMRI (multiparametric MRI) were compared with Friedman's two- 

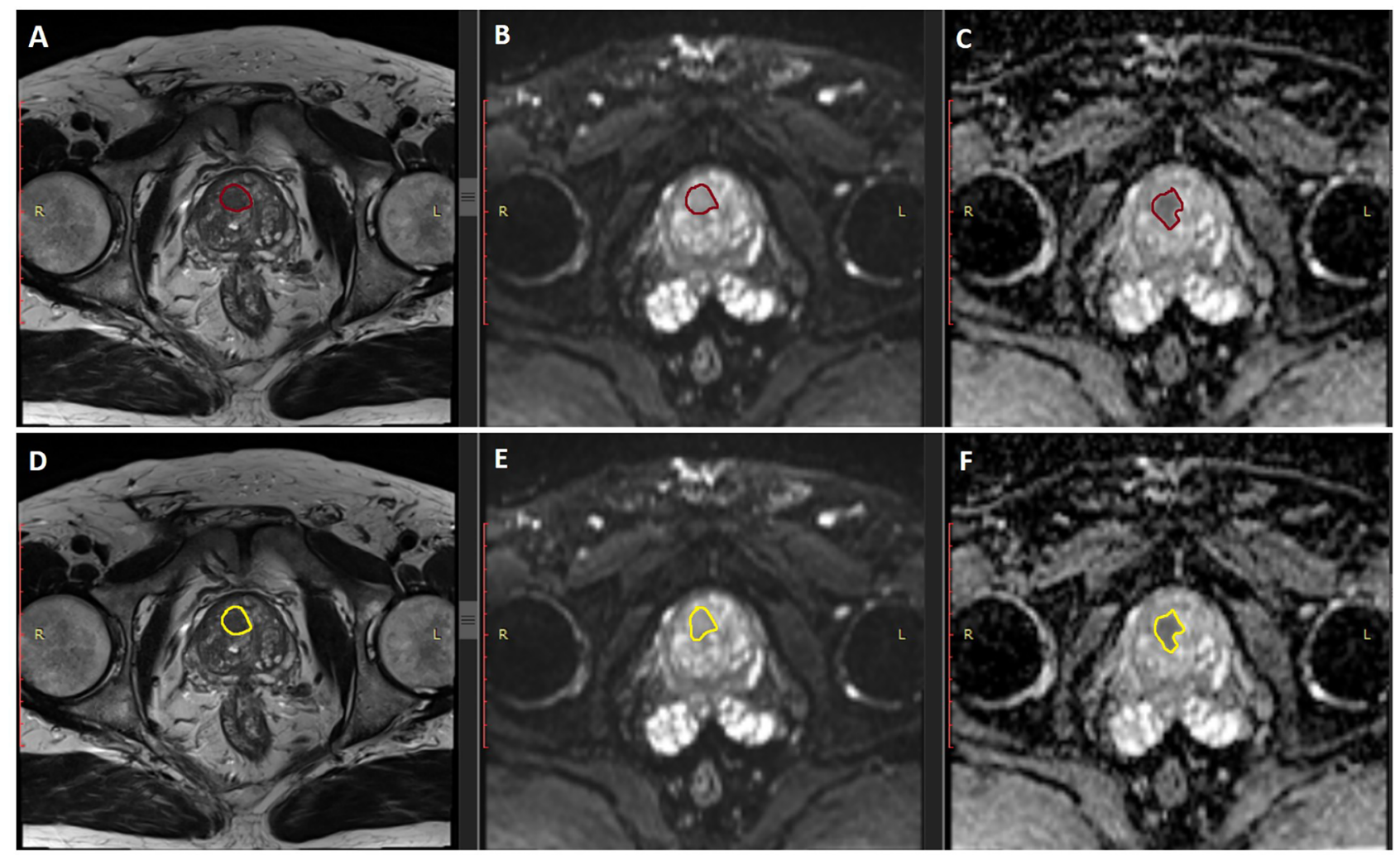

FIGURE 1 | Examples of the two segmentation of the two operators. Panels (A-C) represent the examples of segmentation (in burgundy) of the first operator, in the T2, DWI. and ADC MRI sequences, respectively. Panels (D-F) represent the examples of segmentation (in yellow) of the second operator, in the same sequences, respectively.

way analysis of variance by ranks and with the Wilcoxon signed-rank test. We considered as statistically significant a $p$-value $<0.05$. All the statistical analysis was calculated using SPSS statistical package version 23 (SPSS Inc., Chicago, IL, USA).

\section{RESULTS}

\section{Population}

Forty-four consecutive patients were included in the present analysis. In 26 patients (59.1\%), the prostate biopsy confirmed the presence of prostate cancer, which was scored as Gleason 6 in 6 patients (13.6\%), Gleason $3+4$ in 8 patients $(18.2 \%)$, and Gleason $4+3$ in 12 patients $(27.3 \%)$.

\section{Reliability of Texture Features}

The reliability of texture parameters across different MRI acquisition has shown poor reliability among the two operators in a significant percentage of patients (see Table 2).

Specifically, ICC was scored as poor in $74 \%$ of T2, in $22 \%$ of ADC, in $82 \%$ of DWI50, in $54 \%$ of DWI400, and in $86 \%$ of DWI1,500 (see Figure 2). The ADC sequences showed scores with better reliability, with $33 \%$ of the texture parameters scored as good and excellent ICC. The median values of ICC were, respectively, $0.25 \pm 0.53$ (T2), $0.49 \pm 0.77$ (ADC), $0.11 \pm 0.92$ (DWI50), $0.47 \pm 0.33$ (DWI400), and $0.12 \pm 0.93$ (DWI1,500).

\section{Correlation of ICC With MRI Sequences and Radiomics Analysis Subsections}

The distribution of ICC across the different subsections of radiomics analysis in the different MRI sequences was significantly different in histogram ( $p$ : 0.046), GLCM ( $p$ : 0.001), GLRLM ( $p: 0.014)$, and GLZLM features ( $p: 0.004)$ and was the same in NGLDM ( $p: 0.066)$ and shape features ( $p$ : 0.326) (see Figure 3).

Considering the ADC and the DWI400 sequences as the most reliable sequences, there were significant differences in the distribution of ICC in GLCM features ( $p$ : 0.018 ), with no differences in the other subsections of shape features ( $p$ : 0.893), GLRLM ( $p: 0.594)$, NGLDM ( $p: 0.109)$, and GLZLM ( $p: 0.594)$ (see Figure 4).

\section{DISCUSSION}

Prostate cancer involves tumors with different biological patterns and characteristics and, consequently, different prognoses. The 
TABLE 1 | Texture analysis parameters calculated with the LIFEx software and the corresponding description.

Type of radiomics feature
Radiomics feature name

Description

Homogeneity

Energy

Correlation

Contrast

Entropy

Dissimilarity

Gray-level run length matrix (GLRLM): gives the size of homogeneous runs for each gray SRE (short-run level

emphasis)
LRE (long-run emphasis)

LGRE (low gray-level run emphasis)

HGRE (high gray-level

run emphasis)

SRLGE (short-run low

gray-level emphasis)

SRHGE (short-run high

gray-level emphasis)

LRLGE (long-run low

gray-level emphasis)

LRHGE (long-run high

gray-level emphasis)

GLNUr (gray-level non-

uniformity for run

RLNU (run-length non-

uniformity)

$\mathrm{RP}$ (run percentage)

Neighborhood gray-level different matrix (NGLDM): corresponds to the difference of gray Coarseness

level between one voxel and its 26 neighborhoods in three dimensions

Gray-level zone length matrix (GLZLM): provides information on the size of

homogeneous zones for each gray level in three dimensions

Indices from sphericity

\section{Contrast}

Busyness

SZE (short-zone

emphasis)

LZE (long-zone

emphasis)

LGZE (low gray-level

zone emphasis)

HGZE (high gray-level

zone emphasis)

SZLGE (short-zone low

gray-level emphasis)

SZHGE (short-zone high

gray-level emphasis)

LZLGE (long-zone low

gray-level emphasis)

LZHGE (long-zone high

gray-level emphasis)

GLNUz (gray-level non-

uniformity for zone)

RLNU (zone length nonuniformity)

ZP (zone percentage)

Sphericity

Volume (ml or vx)

Surface

Compacity

Skewness

Kurtosis

Min

Max
Homogeneity of gray-level voxel pairs Uniformity of gray-level voxel pairs

Linear dependency of gray levels in GLCM

Local variations in the GLCM

Randomness of gray-level voxel pairs

Variation of gray-level voxel pairs

Distribution of the short homogeneous runs in an image

Distribution of the long homogeneous runs in an image

Distribution of the low gray-level runs

Distribution of the high gray-level runs

Distribution of the short homogeneous runs with low gray levels

Distribution of the short homogeneous runs with high gray levels

Distribution of the long homogeneous runs with low gray levels

Distribution of the long homogeneous runs with high gray levels

Non-uniformity of the gray levels of the

homogeneous runs

Length of the homogeneous runs

Homogeneity of the homogeneous runs Level of spatial rate of change in intensity Intensity difference between neighboring regions

Spatial frequency of changes in intensity

Distribution of the short homogeneous zones in an image

Distribution of the long homogeneous zones in an image

Distribution of the low gray-level zones

Distribution of the high gray-level zones

Distribution of the short homogeneous zones with low gray levels

Distribution of the short homogeneous zones with high gray levels

Distribution of the long homogeneous zones with low gray levels

Distribution of the long homogeneous zones with high gray levels

Non-uniformity of the gray levels of the homogeneous zones

Length of the homogeneous runs

Homogeneity of the homogeneous zones Measures how spherical a volume of interest is Measures the volume in voxels or milliliter Measures the surface of the volume of interest Measures the degree to which the volume of interest is compact

Measures the asymmetry of the gray-level distribution in the histogram

Measures whether the gray-level distribution is peaked or flat relative to a normal distribution Measures the minimal value of Hounsfield unit Measures the maximal value of Hounsfield unit 
TABLE 1 | Continued

Type of radiomics feature

Radiomics feature

name

Mean

Std
Measures the mean value of Hounsfield unit

Measures the standard deviation of the distribution of Hounsfield unit histogram therapeutic approach is a field of fervent debate especially in localized stages, with different strategies being adopted $(22,23)$.

A deeper knowledge of the specific biological pattern is needed to provide the correct treatment in each PCa patient. Biological and molecular analysis had already been correlated with patterns of disease, and nowadays, radiomics represents an increasingly interesting frontier in the oncology field $(10,24)$. However, there are still many limitations for the analysis of quantitative

TABLE 2 | Intraclass coefficient correlation (ICC) of the different texture features in the different mrMRI sequences.

\begin{tabular}{|c|c|c|c|c|c|}
\hline Parameter & T2 & ADC & DWI 50 & DWI 400 & DWI 1,500 \\
\hline HIST_min & 0.573 & 0.658 & 0.346 & 0.593 & 0.113 \\
\hline HIST_mean & 0.723 & 0.637 & 0.317 & 0.807 & 0.127 \\
\hline HIST_std & 0.644 & 0.440 & 0.070 & 0.596 & 0.151 \\
\hline HIST_max & 0.749 & 0.587 & 0.164 & 0.675 & 0.204 \\
\hline HIST_Skewness & 0.170 & 0.499 & 0.395 & 0.299 & -0.273 \\
\hline HIST_Kurtosis & 0.302 & 0.542 & 0.299 & -0.131 & 0.397 \\
\hline SHAPE_Volume.ml & 0.328 & 0.106 & 0.118 & 0.308 & 0.87 \\
\hline SHAPE_Volume.vx & 0.365 & 0.313 & 0.196 & 0.192 & 0.111 \\
\hline SHAPE_Sphericity & 0.025 & 0.022 & 0.067 & 0.155 & -0.310 \\
\hline SHAPE_Surface & 0.201 & 0.736 & -1.282 & 0.524 & 0.004 \\
\hline SHAPE_Compacity & 0.076 & 0.611 & -1.480 & 0.700 & -0.114 \\
\hline GLCM_Homogeneity & 0.559 & 0.920 & 0.713 & 0.710 & 0.841 \\
\hline GLCM_Energy & -0.860 & 0.187 & -0.238 & 0.060 & -1.554 \\
\hline GLCM_Contrast & 0.601 & 0.868 & 0.395 & 0.684 & 0.066 \\
\hline GLCM_Correlation & 0.286 & 0.910 & 0.088 & 0.728 & 0.620 \\
\hline GLCM_Entropy_log10 & -1.736 & 0.790 & -3.527 & 0.218 & -1.478 \\
\hline GLCM_Entropy_log2 & -1.736 & 0.790 & -3.527 & 0.218 & -1.478 \\
\hline GLCM_Dissimilarity & 0.630 & 0.926 & 0.620 & 0.741 & 0.482 \\
\hline GLRLM_SRE & 0.378 & 0.295 & 0.535 & 0.717 & 0.626 \\
\hline GLRLM_LRE & 0.374 & 0.393 & 0.608 & 0.571 & 0.391 \\
\hline GLRLM_LGRE & -0.209 & -0.245 & -0.100 & 0.076 & -0.320 \\
\hline GLRLM_HGRE & -0.058 & 0.801 & -1.219 & 0.189 & -2.259 \\
\hline GLRLM_SRLGE & -0.201 & -0.228 & -0.098 & 0.088 & -0.255 \\
\hline GLRLM_SRHGE & -0.065 & 0.807 & -0.784 & 0.143 & -1.719 \\
\hline GLRLM_LRLGE & -0.247 & -0.322 & -0.115 & 0.041 & -0.492 \\
\hline GLRLM_LRHGE & -0.025 & 0.771 & -0.806 & 0.594 & -5.126 \\
\hline GLRLM_GLNU & 0.546 & 0.347 & 0.608 & 0.496 & 0.015 \\
\hline GLRLM_RLNU & 0.310 & 0.383 & 0.041 & 0.102 & 0.127 \\
\hline GLRLM_RP & 0.391 & 0.379 & 0.562 & 0.678 & 0.639 \\
\hline NGLDM_Coarseness & 0.125 & 0.528 & 0.515 & 0.795 & 0.113 \\
\hline NGLDM_Contrast & 0.241 & 0.101 & -0.023 & 0.170 & -0.892 \\
\hline NGLDM_Busyness & 0.560 & 0.137 & 0.272 & 0.702 & -0.057 \\
\hline GLZLM_SZE & 0.513 & 0.789 & 0.427 & 0.863 & 0.433 \\
\hline GLZLM_LZE & 0.257 & 0.433 & 0.410 & 0.952 & 0.000 \\
\hline GLZLM_LGZE & -0.246 & -0.120 & -0.141 & 0.101 & -0.276 \\
\hline GLZLM_HGZE & -0.146 & 0.827 & -0.836 & -0.185 & -0.816 \\
\hline GLZLM_SZLGE & -0.165 & 0.039 & -0.110 & 0.214 & 0.345 \\
\hline GLZLM_SZHGE & 0.011 & 0.877 & 0.535 & -0.111 & -0.152 \\
\hline GLZLM_LZLGE & 0.124 & -4.067 & 0.417 & -0.104 & -0.011 \\
\hline GLZLM_LZHGE & 0.355 & 0.179 & 0.106 & 0.972 & 0.000 \\
\hline GLZLM_GLNU & 0.332 & 0.481 & 0.285 & 0.470 & 0.284 \\
\hline GLZLM_ZLNU & 0.003 & 0.637 & -0.443 & 0.106 & -0.241 \\
\hline GLZLM_ZP & 0.669 & 0.811 & 0.477 & 0.916 & 0.656 \\
\hline Poor reliability $(\mathrm{ICC}<0.5)$ & $32(74 \%)$ & $22(51 \%)$ & $35(82 \%)$ & $23(54 \%)$ & $37(86 \%)$ \\
\hline Moderate reliability (ICC 0.5-0.75) & $11(26 \%)$ & $7(16 \%)$ & $8(18 \%)$ & $14(32 \%)$ & $4(9 \%)$ \\
\hline Good reliability (ICC 0.75-0.9) & 0 & $11(26 \%)$ & 0 & $3(7 \%)$ & $2(5 \%)$ \\
\hline Excellent reliability (ICC > 0.9) & 0 & $3(7 \%)$ & 0 & $3(7 \%)$ & 0 \\
\hline
\end{tabular}

In bold the classification of the texture features. 


\section{ICC distribution}

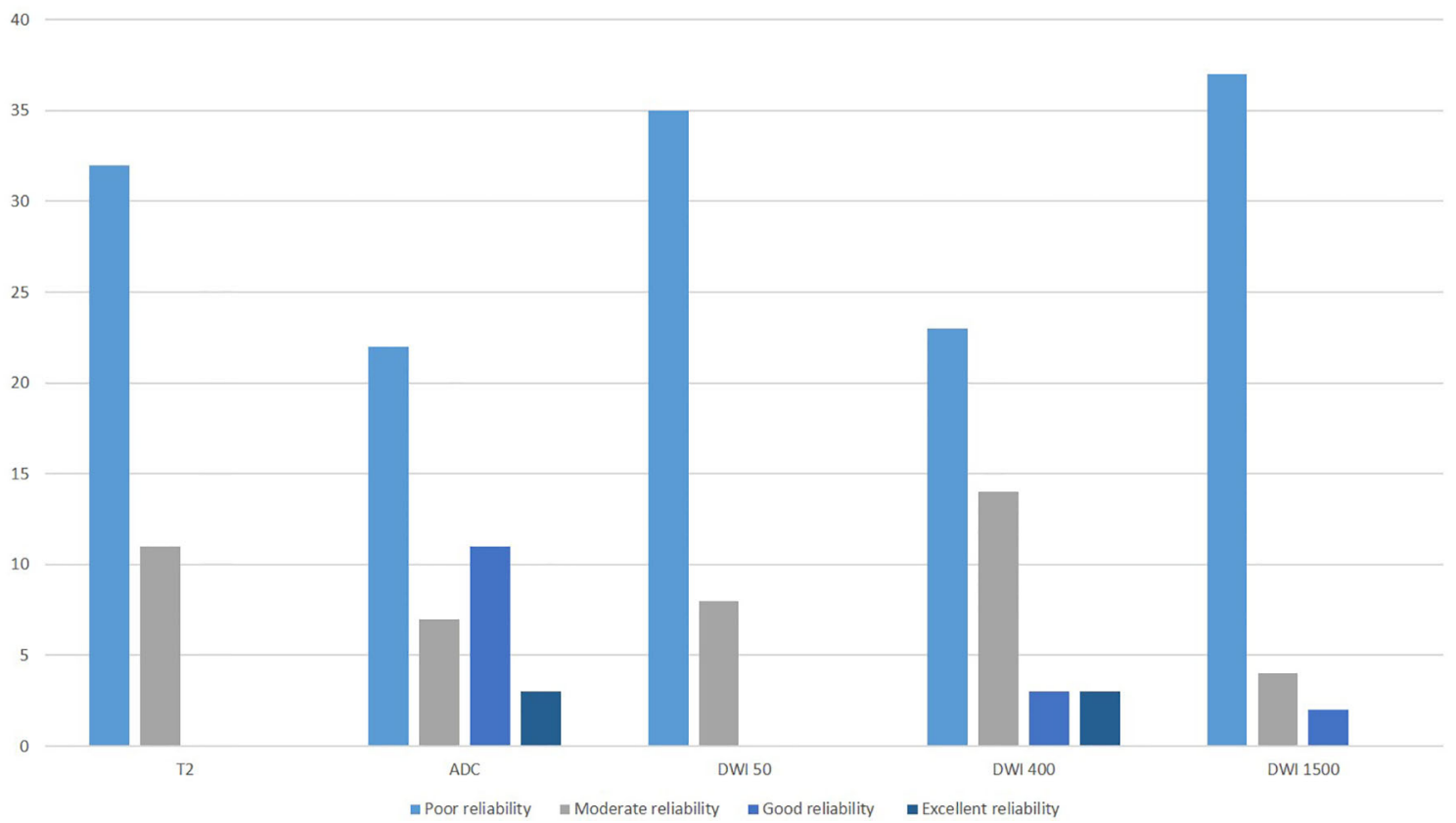

FIGURE 2 | Intraclass coefficient correlation (ICC) distribution of texture features across the different mpMRI imaging acquisitions.

parameters through dedicated software to enter the routine diagnostic pipeline, relying on the reproducibility of collected data. MpMRI is routinely used as part of the diagnostic workup and staging algorithm in PCa (25). In fact, recent studies suggest that mpMRI reduces the number of unnecessary prostate biopsies in patients with PI-RADS score of 3 or more. T2, DWI, and ADC are the most commonly employed sequences assessing PI-RADS score. ADC could heavily support clinical workflow in decisionmaking for patients with PI-RADS score $<3$ who are considered at risk for PCa $(26,27)$.

Thus, the implementation and validation of radiomic features is seemingly feasible and convenient, to gather more information

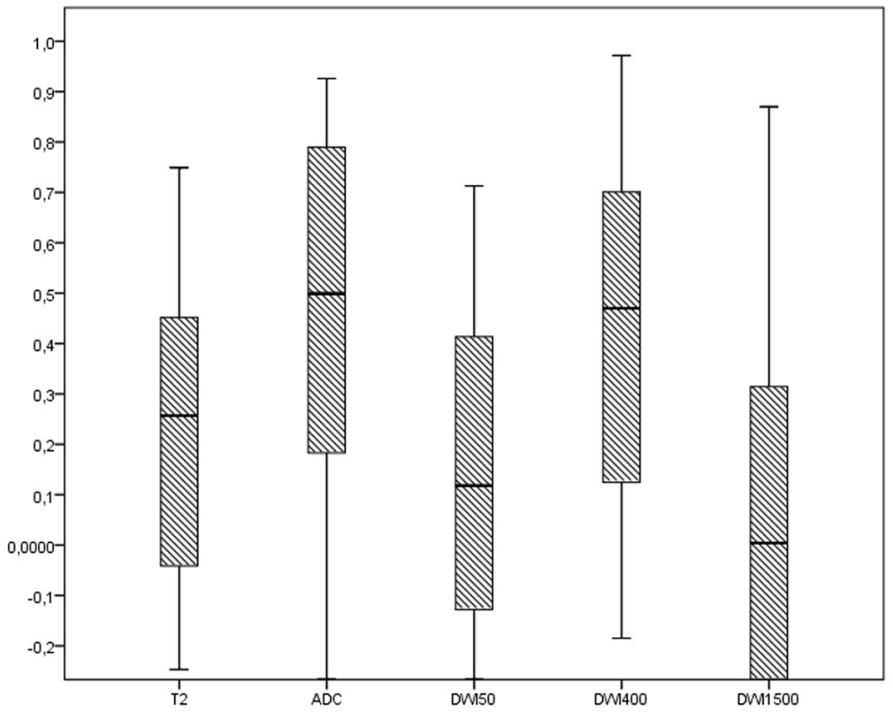

FIGURE 3 | The distribution of ICC of radiomics features across the different MRI sequences. 

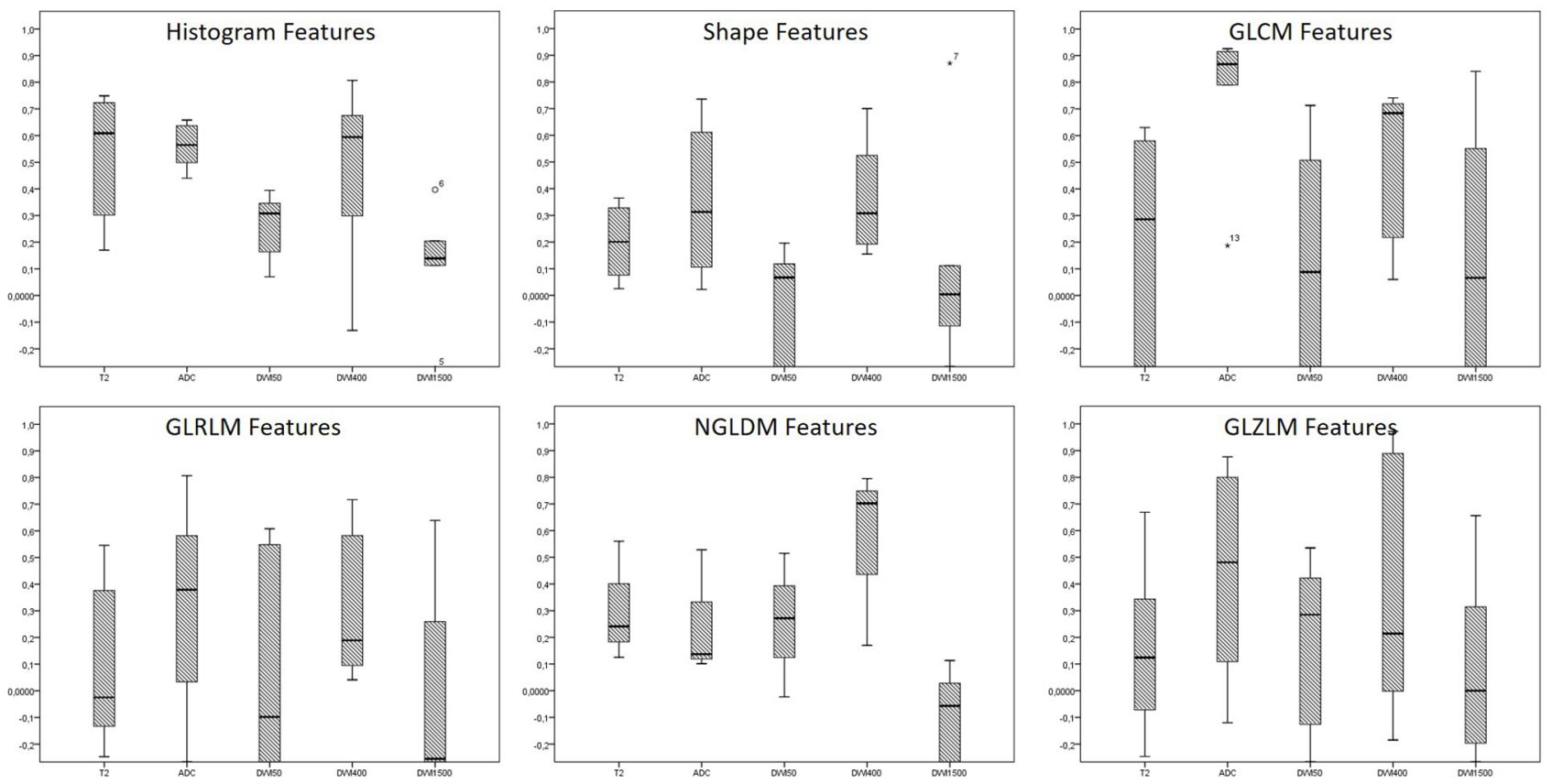

FIGURE 4 | The distribution of ICC across the different subclasses of radiomics features in the different MRI sequences. * are the outliers.

with limited additional costs as well as avoid invasive diagnostic procedures (e.g., transrectal gland biopsy).

In radiomics, it is of paramount importance to understand that most of the features were originally developed for non-medical imaging and for planar images. In the context of clinical investigation, the final aim of radiomics is to use texture features as surrogate biomarkers of different clinical endpoints. Thus, surrogate biomarkers must be correlated to the endpoint, and at the same time, their mensuration must be accurate and robust $(28,29)$.

Every single process in the workflow of radiomics (image acquisition and reconstruction, segmentation, feature extraction, data analysis, model building, and validation) has its own challenges, and in particular, the challenges relevant to robustness of radiomics features has been analyzed by many researchers in recent years $(30,31)$.

Our study focused on reliability validation of specific radiomic parameters extracted from mpMRI in a diagnostic setting, while recently published reports in the application of radiomics at a therapeutic level investigated radiomics feature reliability carrying out a thorough assessment of repeatability and reproducibility of MRI radiomics features in MRI-guided radiotherapy (MRgRT) in PCa (32). Similar to our results, in this study, the differences originating from MRI acquisition were notably described as the most impactful on reliability (33), and only a few features showed good to excellent repeatability and reproducibility coefficients, although such identification is still insufficient for a reliable radiomics study (32). The observed reliability is poor also in other studies, in accordance with our results $(34,35)$.
Detection of PCa lesions using radiomics feature extracted from mpMRI (36) images and an objective increase in sensitivity and specificity in detecting $\mathrm{PCa}$ (37) with computer-aided diagnosis tools are already possible although non-negligible uncertainties may occur. Moreover, in Gleason and PI-RADS prediction, texture-based features, geometric parameters, and contrast and homogeneity GLCM features in different studies have shown radiomics-augmented capability to predict GS. The future uses of radiomics could involve aftertreatment evaluation of biochemical recurrence risk, to better stratify patients after radical prostatectomy, therefore helping the clinician to adapt postoperative management.

Despite its potential, the low reproducibility of radiomics approaches represents a significant hurdle to enter the routine clinical workup of PCa.

Homogeneous protocols based on radiomics are yet to be developed in both diagnostic and therapeutic settings. The poor reliability of datasets does not allow comparisons between cohorts of different patients.

The causes of low reliability are several and complex to analyze. A fundamental role is played by the experience of the operator, but given the relatively recent development of radiomics, it is rare to identify operators with significant and consistent experience.

Furthermore, the diagnostic assessment of PCa through mpMRI has always been challenging far earlier than the radiomics approach, due to the difficulties and heterogeneity in prostate cancer segmentation and PI-RADS score assignment (38-40). To date, no guidelines are available for contouring prostate cancer inside the gland, as visible on mpMRI, and the 
analysis of the contourings of the multicenter phase III FLAME trial showed significant different interpretations in tumor contouring between institutes (40).

In this regard, sharing images, radiomics feature, and contouring guidelines and the implications derived from them represent an unmet need that must be solved in the near future. Further studies on genomics feature are needed to face the poor reliability issue, empowering the role of radiomics applications for PCa.

\section{LIMITATIONS}

We recognize many limitations in our work. Firstly, we performed a retrospective analysis of a single cohort of homogeneous patients with suspicious prostate cancer. At the same time, the number of analyzed patients is low.

\section{CONCLUSIONS}

In our study, we demonstrated that the reliability of MRI features in prostate cancer is extremely low. These findings reinforce the pivotal importance of preclinical studies before applying radiomics in clinical practice.

\section{REFERENCES}

1. Siegel RL, Miller KD, Fuchs HE, Jemal A. Cancer Statistics, 2021. CA Cancer J Clin (2021) 71(1):7-33. doi: 10.3322/caac.21654

2. Sung H, Ferlay J, Siegel RL, Laversanne M, Soerjomataram I, Jemal A, et al. Global Cancer Statistics 2020: GLOBOCAN Estimates of Incidence and Mortality Worldwide for 36 Cancers in 185 Countries. CA Cancer J Clin (2021) 71(3):209-49. doi: 10.3322/caac.21660

3. Leslie SW, Soon-Sutton TL, Sajjad H, Siref LE. Prostate Cancer. In: StatPearls. Treasure Island (FL): StatPearls Publishing (2021). Copyright (C) 2021, StatPearls Publishing LLC.

4. Epstein JI, Egevad L, Amin MB, Delahunt B, Srigley JR, Humphrey PA. The 2014 International Society of Urological Pathology (ISUP) Consensus Conference on Gleason Grading of Prostatic Carcinoma: Definition of Grading Patterns and Proposal for a New Grading System. Am J Surg Pathol (2016) 40(2):244-52. doi: 10.1097/PAS.0000000000000530

5. Morris LG, Riaz N, Desrichard A, Şenbabaoğlu Y, Hakimi AA, Makarov V, et al. Pan-Cancer Analysis of Intratumor Heterogeneity as a Prognostic Determinant of Survival. Oncotarget (2016) 7(9):10051-63. doi: 10.18632/ oncotarget.7067

6. Liu J, Dang H, Wang XW. The Significance of Intertumor and Intratumor Heterogeneity in Liver Cancer. Exp Mol Med (2018) 50(1):e416. doi: 10.1038/ emm.2017.165

7. Burrell RA, McGranahan N, Bartek J, Swanton C. The Causes and Consequences of Genetic Heterogeneity in Cancer Evolution. Nature (2013) 501(7467):338-45. doi: 10.1038/nature12625

8. Yang F, Wang Y, Li Q, Cao L, Sun Z, Jin J, et al. Intratumor Heterogeneity Predicts Metastasis of Triple-Negative Breast Cancer. Carcinogenesis (2017) 38(9):900-9. doi: 10.1093/carcin/bgx071

9. Haffner MC, Zwart W, Roudier MP, True LD, Nelson WG, Epstein JI, et al. Genomic and Phenotypic Heterogeneity in Prostate Cancer. Nat Rev Urol (2021) 18(2):79-92. doi: 10.1038/s41585-020-00400-w

10. Mayerhoefer ME, Materka A, Langs G, Häggström I, Szczypiński P, Gibbs P, et al. Introduction to Radiomics. J Nucl Med (2020) 61(4):488-95. doi: 10.2967/jnumed.118.222893

\section{DATA AVAILABILITY STATEMENT}

The raw data supporting the conclusions of this article will be made available by the authors, without undue reservation.

\section{ETHICS STATEMENT}

Ethical review and approval was not required for the study on human participants in accordance with the local legislation and institutional requirements. The patients/participants provided their written informed consent to participate in this study.

\section{AUTHOR CONTRIBUTIONS}

$\mathrm{VN}, \mathrm{AR}, \mathrm{FU}$, and $\mathrm{SC}$ conceived and designed the study and wrote the manuscript. VP, AA, LD'A, PR, GR, LG, and MC acquired the clinical data. VN performed the statistical analysis. VN, FU, $\mathrm{CV}, \mathrm{AR}$, and SC followed the patients, including planning clinical visits, blood sample collection, and follow-up. LA, SC, and AR revised the paper. All authors read and approved the submitted version of the manuscript.

11. Reginelli A, Nardone V, Giacobbe G, Belfiore MP, Grassi R, Schettino F, et al. Radiomics as a New Frontier of Imaging for Cancer Prognosis: A Narrative Review. Diagnostics (2021) 11(10):1796. doi: 10.3390/diagnostics11101796

12. Nardone V, Boldrini L. Radiomics in the Setting of Neoadjuvant Radiotherapy: A New Approach for Tailored Treatment. Cancers (2021) 13 (14):3590. doi: 10.3390/cancers 13143590

13. Brunese L, Mercaldo F, Reginelli A, Santone A. Radiomics for Gleason Score Detection Through Deep Learning. Sensors (Switzerland) (2020) 20(18):1-23. doi: 10.3390/s20185411

14. Malayeri AA, El Khouli RH, Zaheer A, Jacobs MA, Corona-Villalobos CP, Kamel IR, et al. Principles and Applications of Diffusion-Weighted Imaging in Cancer Detection, Staging, and Treatment Follow-Up. Radiographics (2011) 31(6):1773-91. doi: 10.1148/rg.316115515

15. Brunese L, Mercaldo F, Reginelli A, Santone A. Formal Methods for Prostate Cancer Gleason Score and Treatment Prediction Using Radiomic Biomarkers. Magn Reson Imaging (2020) 66:165-75. doi: 10.1016/j.mri.2019.08.030

16. Nioche C, Orlhac F, Boughdad S, Reuze S, Goya-Outi J, Robert C, et al. LIFEx: A Freeware for Radiomic Feature Calculation in Multimodality Imaging to Accelerate Advances in the Characterization of Tumor Heterogeneity. Cancer Res (2018) 78(16):4786-9. doi: 10.1158/0008-5472.CAN-18-0125

17. Watson PF, Petrie A. Method Agreement Analysis: A Review of Correct Methodology. Theriogenology (2010) 73(9):1167-79. doi: 10.1016/ j.theriogenology.2010.01.003

18. Portney LG, Watkins MP. Foundations of Clinical Research: Applications to Practice. 2nd edition. PH Health, editor. Upper Saddle River, N.J: Pearson Education Limited (2000).

19. Mazzei MA, Giacomo LD, Bagnacci G, Nardone V, Gentili F, Lucii G, et al. Delta-Radiomics and Response to Neoadjuvant Treatment in Locally Advanced Gastric Cancer-A Multicenter Study of GIRCG (Italian Research Group for Gastric Cancer). Quant Imaging Med Surg (2021) 11 (6):2376-87. doi: 10.21037/qims-20-683

20. Nardone V, Reginelli A, Guida C, Belfiore MP, Biondi M, Mormile M, et al. Delta-Radiomics Increases Multicentre Reproducibility: A Phantom Study. Med Oncol (Northwood London England) (2020) 37(5):38. doi: 10.1007/s12032-02001359-9 
21. Nardone V, Tini P, Croci S, Carbone SF, Sebaste L, Carfagno T, et al. 3D Bone Texture Analysis as a Potential Predictor of Radiation-Induced Insufficiency Fractures. Quant Imaging Med Surg (2018) 8(1):14-24. doi: 10.21037/ qims.2018.02.01

22. Grozescu T, Popa F. Prostate Cancer Between Prognosis and Adequate/Proper Therapy. J Med Life (2017) 10(1):5-12.

23. Nardone V, Botta C, Caraglia M, Martino EC, Ambrosio MR, Carfagno T, et al. Tumor Infiltrating T Lymphocytes Expressing FoxP3, CCR7 or PD-1 Predict the Outcome of Prostate Cancer Patients Subjected to Salvage Radiotherapy After Biochemical Relapse. Cancer Biol Ther (2016) 17 (11):1213-20. doi: 10.1080/15384047.2016.1235666

24. Martinez-Jimenez F, Muinos F, Sentis I, Deu-Pons J, Reyes-Salazar I, ArnedoPac C, et al. A Compendium of Mutational Cancer Driver Genes. Nat Rev Cancer (2020) 20(10):555-72. doi: 10.1038/s41568-020-0290-x

25. Litwin MS, Tan HJ. The Diagnosis and Treatment of Prostate Cancer: A Review. Jama (2017) 317(24):2532-42. doi: 10.1001/jama.2017.7248

26. Pepe P, D'Urso D, Garufi A, Priolo G, Pennisi M, Russo G, et al. Multiparametric MRI Apparent Diffusion Coefficient (ADC) Accuracy in Diagnosing Clinically Significant Prostate Cancer. In Vivo (Athens Greece) (2017) 31(3):415-8. doi: 10.21873/invivo.11075

27. Pepe P, Garufi A, Priolo GD, Galia A, Fraggetta F, Pennisi M. Is it Time to Perform Only Magnetic Resonance Imaging Targeted Cores? Our Experience With 1,032 Men Who Underwent Prostate Biopsy. J Urol (2018) 200(4):7748. doi: 10.1016/j.juro.2018.04.061

28. Bamberg F. Quantitative Imaging and Imaging Biomarkers: The Search for Generalizability in Radiology. J Thorac Imaging (2018) 33(2):69-70. doi: 10.1097/RTI.0000000000000321

29. Raunig DL, McShane LM, Pennello G, Gatsonis C, Carson PL, Voyvodic JT, et al. Quantitative Imaging Biomarkers: A Review of Statistical Methods for Technical Performance Assessment. Stat Methods Med Res (2015) 24(1):2767. doi: 10.1177/0962280214537344

30. Zhao B, Tan Y, Tsai WY, Qi J, Xie C, Lu L, et al. Reproducibility of Radiomics for Deciphering Tumor Phenotype With Imaging. Sci Rep (2016) 6:23428. doi: $10.1038 /$ srep 23428

31. Ganeshan B, Miles KA. Quantifying Tumour Heterogeneity With CT. Cancer Imaging (2013) 13(1):140-9. doi: 10.1102/1470-7330.2013.0015

32. Xue C, Yuan J, Poon DM, Zhou Y, Yang B, Yu SK, et al. Reliability of MRI Radiomics Features in MR-Guided Radiotherapy for Prostate Cancer: Repeatability, Reproducibility, and Within-Subject Agreement. Med Phys (2021) 48(11):6976-86. doi: 10.1002/mp.15232

33. Traverso A, Wee L, Dekker A, Gillies R. Repeatability and Reproducibility of Radiomic Features: A Systematic Review. Int J Radiat Oncol Biol Phys (2018) 102(4):1143-58. doi: 10.1016/j.ijrobp.2018.05.053

34. Dulhanty C, Wang L, Cheng M, Gunraj H. Radiomics Driven Diffusion Weighted Imaging Sensing Strategies for Zone-Level Prostate Cancer
Sensing. Sensors (Basel Switzerland) (2020) 20(5):1539. doi: 10.3390/ s20051539

35. Scalco E, Belfatto A, Mastropietro A, Rancati T, Avuzzi B, Messina A, et al. T2w-MRI Signal Normalization Affects Radiomics Features Reproducibility. Med Phys (2020) 47(4):1680-91. doi: 10.1002/mp.14038

36. Ginsburg SB, Algohary A, Pahwa S, Gulani V, Ponsky L, Aronen HJ, et al. Radiomic Features for Prostate Cancer Detection on MRI Differ Between the Transition and Peripheral Zones: Preliminary Findings From a MultiInstitutional Study. J Magn Reson Imaging (2017) 46(1):184-93. doi: 10.1002/jmri.25562

37. Hambrock T, Vos PC, Hulsbergen-van de Kaa CA, Barentsz JO, Huisman HJ. Prostate Cancer: Computer-Aided Diagnosis With Multiparametric 3-T MR Imaging-Effect on Observer Performance. Radiology (2013) 266(2):521-30. doi: 10.1148/radiol.12111634

38. Oerther B, Engel H, Bamberg F, Sigle A, Gratzke C, Benndorf M. Cancer Detection Rates of the PI-RADSv2.1 Assessment Categories: Systematic Review and Meta-Analysis on Lesion Level and Patient Level. Prostate Cancer Prostatic Dis (2021). doi: 10.1038/s41391-021-00417-1

39. Mehralivand S, Yang D, Harmon SA, Xu D, Xu Z, Roth H, et al. A Cascaded Deep Learning-Based Artificial Intelligence Algorithm for Automated Lesion Detection and Classification on Biparametric Prostate Magnetic Resonance Imaging. Acad Radiol (2021). doi: 10.1016/j.acra.2021.08.019

40. van Schie MA, Dinh CV, Houdt PJV, Pos FJ, Heijmink S, Kerkmeijer LGW, et al. Contouring of Prostate Tumors on Multiparametric MRI: Evaluation of Clinical Delineations in a Multicenter Radiotherapy Trial. Radiother Oncol (2018) 128(2):321-6. doi: 10.1016/j.radonc.2018.04.015

Conflict of Interest: The authors declare that the research was conducted in the absence of any commercial or financial relationships that could be construed as a potential conflict of interest.

Publisher's Note: All claims expressed in this article are solely those of the authors and do not necessarily represent those of their affiliated organizations, or those of the publisher, the editors and the reviewers. Any product that may be evaluated in this article, or claim that may be made by its manufacturer, is not guaranteed or endorsed by the publisher.

Copyright (c) 2021 Urraro, Nardone, Reginelli, Varelli, Angrisani, Patanè, D’Ambrosio, Roccatagliata, Russo, Gallo, De Chiara, Altucci and Cappabianca. This is an open-access article distributed under the terms of the Creative Commons Attribution License (CC BY). The use, distribution or reproduction in other forums is permitted, provided the original author(s) and the copyright owner(s) are credited and that the original publication in this journal is cited, in accordance with accepted academic practice. No use, distribution or reproduction is permitted which does not comply with these terms. 